\title{
The complete chloroplast genome of Primulina and two novel strategies for development of high polymorphic loci for population genetic and phylogenetic studies
}

\author{
Chao Feng ${ }^{1}$, Meizhen $\mathrm{X}^{1,2}$, Chen Feng ${ }^{1,2}$, Eric J. B. von Wettberg ${ }^{3}$ and Ming Kang ${ }^{1 *}$ (D)
}

\begin{abstract}
Background: Primulina Hance is an emerging model for studying evolutionary divergence, adaptation and speciation of the karst flora. However, phylogenetic relationships within the genus have not been resolved due to low variation detected in the cpDNA regions. Chloroplast genomes can provide important information for phylogenetic and population genetic studies. Recent advances in next-generation sequencing (NGS) techniques greatly facilitate sequencing whole chloroplast genomes for multiple individuals. Consequently, novel strategies for development of highly polymorphic loci for population genetic and phylogenetic studies based on NGS data are needed.

Methods: For development of high polymorphic loci for population genetic and phylogenetic studies, two novel strategies are proposed here. The first protocol develops lineage-specific highly variable markers from the true high variation regions (Con_Seas) across whole cp genomes, instead of traditional noncoding regions. The pipeline has been integrated into a single perl script, and named "Con_Sea_Identification_and_PIC_Calculation". The second method assembles chloroplast fragments (poTs) and sub-super-marker (CpContigs) through our "SACRing" pipeline. This approach can fundamentally alter the strategies used in phylogenetic and population genetic studies based on cp markers, facilitating a transition from traditional Sanger sequencing to RAD-Seq. Both of these scripts are available at https:/github. com/scbgfengchao/.

Results: Three complete Primulina chloroplast genomes were assembled from genome survey data, and then two novel strategies were developed to yield highly polymorphic markers. For experimental evaluation of the first protocol, a set of Primulina species were used for PCR amplification. The results showed that these newly developed markers are more variable than traditional ones, and seem to be a better choice for phylogenetic and population studies in Primulina. The second method was also successfully applied in population genetic studies of 21 individuals from three natural populations of Primulina.

Conclusions: These two novel strategies may provide a pathway for similar research in other non-model species. The newly developed high polymorphic loci in this study will promote further the phylogenetic and population genetic studies in Primulina and other genera of the family Gesneriaceae.
\end{abstract}

Keywords: Primulina, Next-generation sequencing, RAD-Seq, Chloroplast assembly, High-variation regions, Sub-super-marker

\footnotetext{
* Correspondence: mingkang@scbg.ac.cn

${ }^{1}$ Key Laboratory of Plant Resources Conservation and Sustainable Utilization,

South China Botanical Garden, Chinese Academy of Sciences, 723 Xingke

Road, Guangzhou 510650, China

Full list of author information is available at the end of the article
} 


\section{Background}

Chloroplast sequences are important molecular tools for studies of plant phylogeny, phylogeography and population genetics [1-4]. Traditionally, selected cpDNA regions have been chosen for analysis, mostly based on their conservation and efficacy in related taxa. By comparing chloroplast genomes of 13 angiosperm lineages, Shaw et al., [5, 6] identified a set of 34 non-coding regions that ranked highest in their potentially informative characters (PIC), an index which is counted by the sum of nucleotide substitutions, indel and inversions between each of two ingroup species and between an ingroup species and an outgroup species. This set of most variable non-coding regions is consequently widely used in plant evolutionary biology and systematics studies. However, recent comparative plastid genomic studies reveal considerable variation and surprisingly little (c. 12-25\%) overlap in the most variable non-coding regions among different lineages [3, 7-10]. Furthermore, around one third of universal barcoding primers were unlikely to work across all the angiosperms [11]. These findings imply that lineage-specific screening is needed for the identification of the most highly variable markers in different clades.

On the other hand, recent advances in next-generation sequencing (NGS) techniques greatly facilitate the sequencing of whole cp genomes for multiple individuals at relatively low cost [12-14]. However, molecular phylogenetic studies of whole chloroplast genome sequences are yet not practical for large clades with hundreds of species, due in part to insufficient capacity to assemble and analyze such large amounts of NGS data. Although several technical innovations have been proposed for cpDNA assembly based on NGS data [15-17], novel strategies aimed at more time-saving, labor-saving and cost-saving are desirable. Paired-end RAD-Seq (restriction-site associated DNA sequencing) [18] could prove to be an efficient tools for obtaining large numbers of partial chloroplast genomes. Owing to the partial cpDNA sequences that RAD-Seq may provide, it can facilitate chloroplast-based phylogenetic reconstruction with high resolution [19-21]. However, to date, methods specific for chloroplast sub-assembly from paired-end RAD-Seq have not been developed.

Primulina Hance is a large genus of the Old World Gesneriaceae with $c$. 170 species that are widely distributed throughout the limestone karst regions of southern China and Southeast Asia, one of the world's biodiversity hotspots [22]. This genus is uniquely suited for studying evolutionary divergence, adaptation and speciation of the karst flora, due to its high species richness and endemism and high degree of habitat specialization [23, 24]. To date, the nuclear ribosomal internal transcribed spacer (ITS) and the plastid non-coding regions trnL-trnF, rpl32-trnL and atpB-rpl32 have been used to reconstruct the relationships of
Primulina [25-27]. These studies provide a phylogenetic framework for the genus. Kang et al., [27] sampled 104 taxa to reconstruct the largest phylogeny of Primulina so far, in which four major clades were resolved. However, phylogenetic relationships among many taxa have not been resolved due to low variation in these cpDNA regions. Recently, genomic resources have been developed for several species by using RNA-Seq [23]. Besides the nuclear genome, the complete chloroplast genome of Primulina can provide important insights into phylogenetic relationship and evolutionary history of this genus.

Here we present three complete chloroplast genomes of Primulina, and two novel strategies to develop highly polymorphic cp markers. The first strategy develops $\mathrm{cp}$ primers from all the highly variable regions (called Con_Seas) across whole cp genome, instead of just traditional noncoding regions. The pipeline has been integrated into a single perl script, and named "Con_Sea_Identification_and_PIC_Calculation". The second strategy uses RADSeq to directly assemble cp fragments (poTs) and subsuper-marker (CpContigs). This second approach represents a fundamental shift away from cp-primer based Sanger sequencing because it creates a reduced cp genome, which could be used for population genetic analysis as well as phylogenetics. The core pipeline, SACRing, was written in bash, combining several popular software tools and our inhouse perl scripts. Both of these scripts are available at https://github.com/scbgfengchao/, and will be continual improved and updated. Here the performance of these methods was evaluated in an experimental dataset.

\section{Methods}

Plant material and DNA extraction

Three individuals each from $P$. linearifolia (population code, GXNN01), P. huaijiensis (GDHJ02), P. eburnea (WHY01) were used for genome survey sequencing and completed chloroplast genome assembly. Forty-nine individuals from 44 Primulina species (Additional file 1: Table S1) were used to evaluate polymorphism of $\mathrm{cp}$ markers developed in this study. In addition, restrictionsite associated DNA sequences (RAD-Seq) of 21 individuals from three natural populations, seven each from $P$. eburnea (CZYX01), P. yongxingensis (CZYX02) and P. juliae (CZYX03), were used for partial cp genome assembly. All leaf samples were frozen in liquid nitrogen and stored at $-80{ }^{\circ} \mathrm{C}$. Total DNA was extracted from the leaves using a modified CTAB method [28] and treated with RNase (TransGen, China).

\section{Library construction, Illumina sequencing and quality control}

For genome survey sequencing, DNA from 3 samples (GXNN01, WHY01 and GDHJ02) was broken into the 
short fragments with the length of $180 \mathrm{bp}, 230 \mathrm{bp}$ and $230 \mathrm{bp}$, respectively, using focused-ultrasonicators (Covaris, USA). Two libraries were built for each sample, and further sequenced by paired-end sequencing technology of Illumina HiSeq $2000^{\mathrm{mm}}$. For RAD-Seq of the three populations, DNA was first treated with restriction enzyme EcoR I (Takara, China), and then several standard steps were performed, from the addition of sequencing adapters, interruption of enzyme digestion products, to break into smaller random pieces, and repairing the end based on existing protocols [18]. Finally, fragments with lengths ranging from 200 to $800 \mathrm{bp}$ were separated on an agarose gel and selected for PCR amplification as sequencing templates. These libraries were sequenced by PE 100 model on Illumina HiSeq $2000^{\mathrm{mm}}$. The programs of library construction and Illumina sequencing was performed by staff of Novogene Bioinformatics Institute (Beijing, China).

The raw reads were first filtered by removing the adapter sequences and low quality sequences using Software FASTX_Toolkit (http://hannonlab.cshl.edu/ fastx_toolkit/index.html) and our in-house perl scripts. Only the reads with a Q20 percentage (i.e., the percentage of sequences with sequencing error rate lower than $1 \%$ ) over $90 \%$ and $\mathrm{N}$ percentage (i.e., the percentage of nucleotides in read which could not be sequenced) less than $5 \%$, were marked as clean data and used for further analysis. The sub-routine above was integrate into the pipeline, and named QC_pe (Additional file 2: File S1), which is available at https://github.com/scbgfengchao/.

\section{Complete chloroplast assembly from genome survey data}

$P$. linearifolia was used for the first pass of genome survey sequencing and complete chloroplast genome assembly. Briefly, we used the software of Bowtie2 (version 2.2.5) [29] with the parameter "-I 150 -X 1000 -nomixed -no-discordant". The cp data of $P$. linearifolia was isolated by mapping it to the cp genome of Boea hygrometrica (GenBank accession id: NC_016468), a closely related species belonging to the Gesneriaceae family with a publically available cp genome sequences [30]. Furthermore, the cp genome of $P$. linearifolia was sub-assembled using the Velvet software (version 1.2.10) [31], with parameter hash_length (kmer_length) setting from 29 to 99 . The sub-assembly with longest contig N50 was selected and further scaffolding based on the original cp data, using SSPACE software (version 3.0) [32] with default parameter $(-\mathrm{m} 32$-o 20 -r 0.9). After that, each scaffold was located against the cp genome of Boea hygrometrica using blat software [33]. The gaps between scaffolds were closed by PCR amplifications and Sanger sequencing. Meanwhile, Sanger sequences were used for nucleotide confirmation, especially at LSC/IR/ SSC boundaries.
The complete cp genomes of P. eburnea and P. huaijiensis were obtained as described above in turn. The difference is that the cp genome of $P$. linearifolia, instead of B. hygrometrica, was used as reference genome to isolate the cp data, and the upper limit of hash_length was set as 125 in Velvet software. Finally, in order to verify the accuracy of the assembled cp genomes, the cp data of the three Primulina species was mapped back to their respective cp genome by using the program SAMtools (version 0.1.19) [34]. The mapping results were displayed with IGV software (version 2.3.57) [35].

\section{Genome annotation and sequence alignment}

The cp genomes of B. hygrometrica and the three Primulina species were annotated using the online program DOGMA (http://dogma.ccbb.utexas.edu/) [36], and modified by detailed manual corrections. The tRNA boundaries and splice sites were modified by using tRNAscan-SE software (version 1.3.1) [37]. The annotated cp genome maps were drawn by using the online OrganellarGenomeDRAW tool (OGDRAW) [38] and local software Circos (version 0.67) [39].

A consensus sequence was obtained by clustalW alignment of the $4 \mathrm{cp}$ genomes (B. hygrometrica and three Primulina species) with manual corrections, especially in the area of exon boundaries. Meanwhile, the correspondence between each cp genome and consensus sequence was built using our in-house perl script (Additional file 3: File S2; also available at https://github.com/scbgfengchao/). Furthermore, the orthologous coding exons (defined as syntenic coding loci) and orthologous noncoding intergenic regions/introns (defined as syntenic noncoding loci) among 4 cp genomes were classified and identified.

\section{Determination of Con_Sea regions and calculation of polymorphic index}

Based on the alignment results, conserved sites among the $4 \mathrm{cp}$ genomes were labeled. "Con_Islands" (defined as regions containing over 50 continuous conserved sites in the cross-genus consensus genome sequences) were first identified, while the regions between two adjacent Con_Islands were named "Con_Seas".

The potentially informative characters (PICs), an index counted by the sum of SNP and Indel between two chloroplast genomes, was used to evaluate the polymorphism in each Con_Sea region. The pipeline above has been integrated into a single perl script, and named "Con_Sea_Identification_and_PIC_Calculation" (https:// github.com/scbgfengchao/, Additional file 4: File S3). The parameter "minimum length of Con_Island" (50 in this study) can be varied. There is no other software dependency for this script, so it targeted toward researchers without bioinformatics background. PIC at each Con_Sea was 
divided into intrageneric and intergeneric levels. Intrageneric PIC was calculated from the average PIC values of pairwise of 3 Primulina species, while intergeneric PIC were equal to the mean of PIC value of 3 groups between each Primulina species and B. hygrometrica.

In addition, the intra-population (CZYX01, CZYX02 and CZYX03) PIC values were calculated based on the average PIC of 21 pairs from 7 individuals of respective populations, respectively, while the PIC information for the interpopulation pairs were counted by the mean of 49 pairs. These calculations were performed with our in-house perl script "PIC_calculation" (Additional file 5: File S4), and it's also available at https://github.com/scbgfengchao/.

\section{Determination of high variation regions and development of chloroplast markers}

The intrageneric and intergeneric polymorphisms (PICs) were analyzed using three different methods of dividing genomic regions. The first method is a sliding window analysis along the consensus sequence, setting the window and step sizes as 100 and $25 \mathrm{bp}$, respectively, then PIC in each window was plotted with the software Circos (version 0.67) [39]. The second and third method is based on gene regions (syntenic coding regions and syntenic noncoding regions) and variable regions (Con_Seas), respectively. The method based on noncoding regions is a traditional strategy to choose highly polymorphic regions, while the third one is a novel strategy first proposed here, and it is a more effective and directed method to screen lineage-specific high variation regions. The PIC in each region, including syntenic noncoding regions and Con_Seas, was displayed with OriginLab Origin (version 8.0) (Microcal Software INc., Northampton, MA, USA). It was worth noting that the length of these regions varies greatly. In general, Sanger sequencing has been widely used to obtain more sequence data from more species or individuals based on polymorphism chloroplast primers, which were developed from high variation regions. Reserving around $100 \mathrm{bp}$ for primer designing, 700 and $1500 \mathrm{bp}$ are effective lengths of single and two-directional Sanger sequencing reactions, respectively. So in this study, these two lengths are considered as key bounds to evaluate fairly the polymorphic degree of regions with different length.

In order to evaluate the efficiency of the identified high polymorphism regions, eight new genus-specific $\mathrm{cp}$ markers, developed from high variation regions based on the Con_Seas method, were tested in the 49 individuals from 44 Primulina species. For comparison, these individuals were also amplified with four traditional cp markers (atpB-rbcL, rpl32-trnL, trnL-trnF and trnS-trnG), which were selected from 19 traditional universal cp primers that have been used in our previous phylogenetic work [27]. It is worth noting that some new $\mathrm{cp}$ primers were designed from slighter lower variation regions, in order to provide a more comprehensive comparison among markers.

\section{Sub-assembly of chloroplast from PE RAD-Seq (SACRing)}

A novel pipeline, SACRing, was performed for subassembly of chloroplast sequences from PE RAD-Seq. This pipeline (Additional file 6: File S5) was written in Bash, combing several in-house perl scripts with mainstream software, such as Bowtie (version 1.1.2) [40], Bowtie2 (version 2.2.5) [29], Stacks (version 1.40) [41], CAP3 [42] and blat [33]. The pipeline is publically available at https://github.com/scbgfengchao/, together with the usage, examples, notes and description of output files, with the schematic shown in Fig. 1. In this study, for evaluating this pipeline, RAD-Seq data of 21 individuals from three Primulina populations were used for sub-assembly of cp genome, and the cp genome of $P$. eburnea was set as the reference.

First, paired-end RAD-Seq data was separated in different samples according to adapters (Fig. 1a). Following quality control (Additional file 7: Table S5, https:// github.com/scbgfengchao/) and previous analysis (Step 1 in SACRing), the cp-related data was extracted from PE RAD-Seq based on mapping results (Step 2 in SACRing). And then, Step 3, the core step, was used for basic clustering and assembly. Hundreds of thousands of Read1 were mapped back to the specific regions of Ref and clustered into several Tag1s. While hundreds or thousands of Read2, which belonged to the same Tag1, were further assembled into a longer Tag2. This step is the most rate-limiting one, so multi-core CPU scripts were designed to linearly shorten the run time. Step 4 was used to constantly and continually assemble for a better result. Tag1s at the same position were mixed into one sequence with the name of paired tags (pTs). Meanwhile, Tag1 and its paired Tag2 were assembled in a contig, and named overlap tags (oTs). If both Tag1s of pT has an overlap with their paired Tag2s, a longer contig was generated, and defined as poTs, i.e., short for paired \& overlap tags. In addition, several longer contigs (defined as CpContigs) were assembled according to overlap information. And a scaffold (sub-super-marker) was further generated according to the position information, replacing the unknown bases with '-' (Fig. 1b).

\section{Population genetic analysis of chloroplast fragments}

Based on the SACRing pipeline, poTs and CpContigs were obtained independently from RAD-Seq data from 21 individuals. For poTs, the most conserved ones, appearing in all the individuals, were selected, and further used for isolation of consistent sequences. Moreover, using CAP3 [42], these sequences were assembled into $\mathrm{cP}$ fragments (Fig. 1c) and shown with Circos (version 0.67) [39]. These cp fragments were then concatenated for 

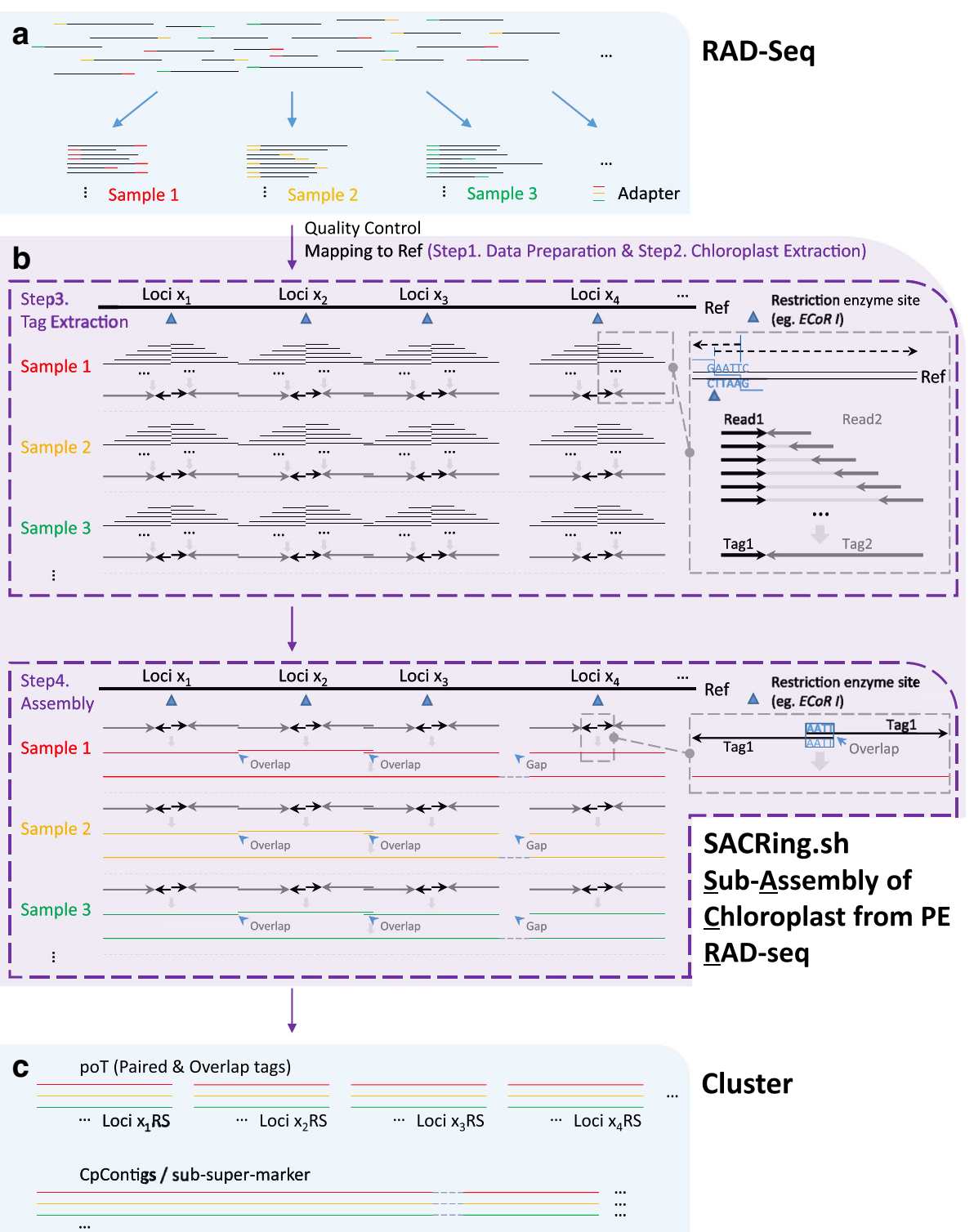

Cluster

Fig. 1 Schematic of sub-assembly of chloroplast genome from PE RAD-seq. a Illustration of RAD-seq method. b Pipeline for sub-assembly of cpDNA from PE RAD-seq. This pipeline, SACRing, was divided into 4 steps, and step 3 and 4 were highlighted in top and bottom boxes with a purple dashed line, respectively. The detail of assembly was enlarged with the boxes with a grey dashed line. $\mathbf{c}$ lllustration of cluster analysis based on the result of SACRing

population genetic analysis. The best substitution model was determined using software jModelTest (version 2.1.7) [43], and then the Bayesian phylogenetic tree was generated with MrBayes (version 3.2.6) [44] under the model of "GTR + I", while Maximum Likelihood phylogenetic tree was performed using RAxML (version 8.2.9) [45] with 1000 replicates under the model of "GTR + I + G", for model "GTR + I + G" is the second best model for cp fragments (poTs), and model "GTR + I" is not supported in RAxML software. The basic indexes of population genetics, such as nucleotide diversity $(\pi)$, theta $(\theta)$ and the average nucleotide diversity between populations $\left(F_{\mathrm{ST}}\right)$, were calculated by DnaSP (version 5.10.1) [46].
Meanwhile, conserved CpContigs of these individuals were also obtained and analyzed as a poTs dataset. In this dataset, "GTR + I + G" was the best substitution model for the construction of a Bayesian phylogenetic tree and a Maximum Likelihood phylogenetic tree.

\section{Results}

Complete chloroplast genome assembly and genomic organization

Using high-throughput genome survey sequencing, we obtained very deep sequence coverage for P. eburnea (population code, WHY01), P. huaijiensis (GDHJ02) and $P$. linearifolia (GXNN01), ranging from 3.1 to 
Table 1 The basic information of genome survey data of the three Primulina species related to chloroplast genomes

\begin{tabular}{lllllll}
\hline Species & Population code & Reads No. $(\mathrm{M})^{\mathrm{a}}$ & ${\text { Throughput }(\mathrm{G})^{\mathrm{b}}}$ & Quality Q30 $^{\mathrm{c}}$ & Cp Size (bp) & Coverage \\
\hline P. eburnea & WHY01 & 12.5 & 3.14 & $97.2 ; 96.9$ & 152,373 & 20,585 \\
P. huaijiensis & GDHJ02 & 18.5 & 4.63 & $97.8 ; 97.5$ & 153,401 & 30,191 \\
P. linearifolia & GXNN01 & 21.1 & 4.22 & $95.4 ; 95.4$ & 153,493 & 27,517 \\
\hline
\end{tabular}

${ }^{\mathrm{a}}$ Reads No. was counted based on the Reads which was used in assembly of cp genomes, instead of whole genome survey sequencing data

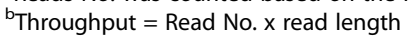

'Quality Q30 was counted by Read1 and Read2 respectively

4.6 G, with Q30 over 95\% in all three species (Table 1). Then, three complete Primulina cp genomes were assembled, with the average coverage between 20,000 and $30,000 \mathrm{x}$, and the minimum of $2000 \mathrm{x}$ (Table 1 and Additional file 8: Fig. S1). No SNPs or Indels were identified when mapping the sequencing data back to their respective $\mathrm{cp}$ genomes, respectively, indicating that the assembled cp genomes are accurate and high-quality. The full length cp genome of $P$. huaijiensis $(153,401 \mathrm{bp})$ is longest and has the longest SC regions (but shortest IR regions), followed with $P$. linearifolia (153,244 bp) and P. eburnea (152,373 bp, as well as shortest SC regions, but longest IR regions) (Fig. 2).

The number and order of predicted functional genes are perfectly consistent among cp genomes of Boea hygrometrica and the three Primulina species (Fig. 2; Additional file 9: File S7 and Additional file 10: Table S2). The cp genome encodes 132 functional genes, with 18 duplicated in the IR regions. Meanwhile, 88 protein coding, 36 tRNA and 8 rRNA genes were identified.

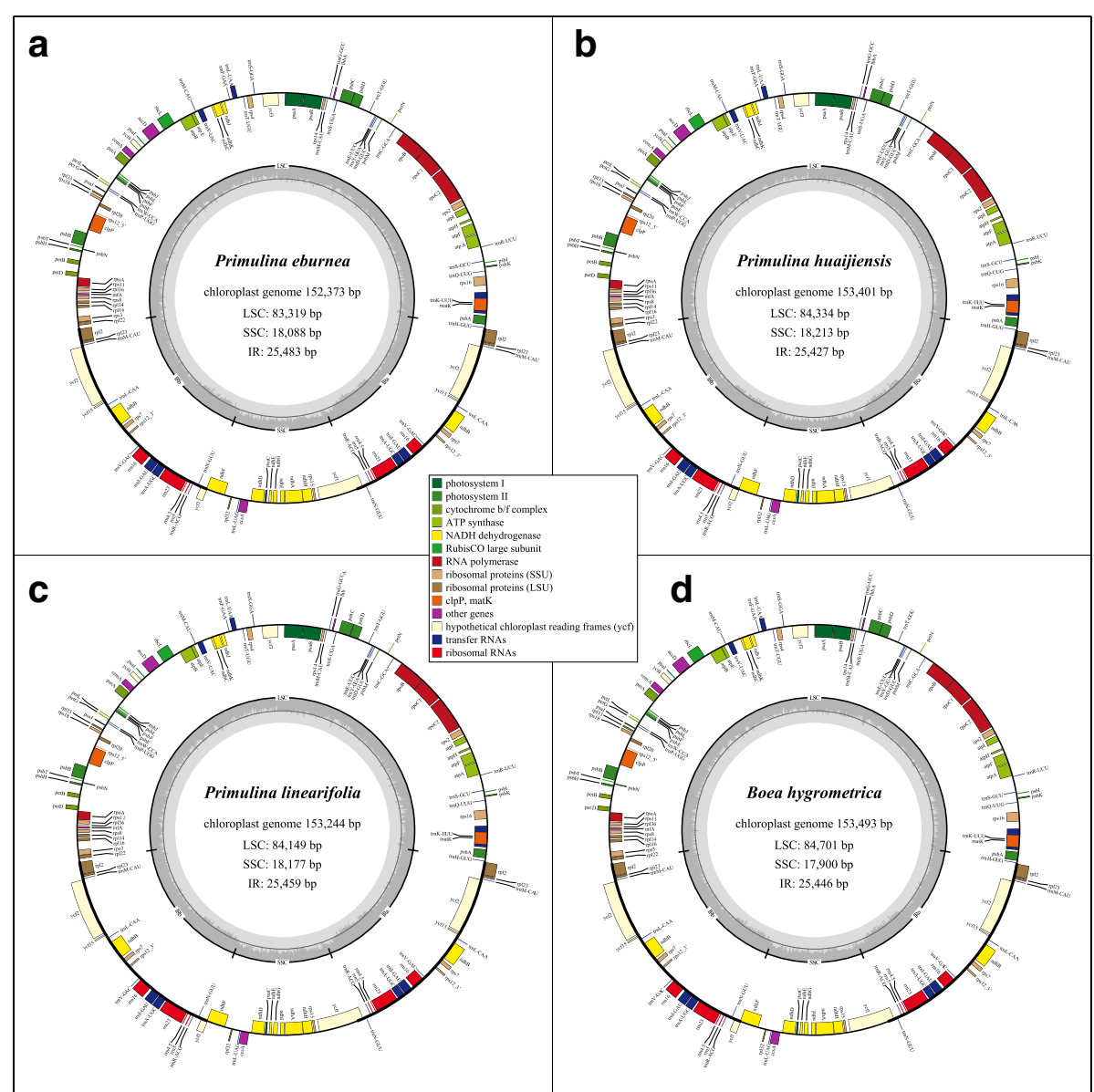

Fig. 2 Circular map of chloroplast genomes of three Primulina species and Boea hygrometrica. Genes shown inside and outside of the outer circle are transcribed clockwise and counterclockwise, respectively. Genes belonging to different groups are marked with different color. The distribution of GC content was shown in the inner circle. Circular map of cp genomes of P. eburnea (a), P. huaijiensis (b), P. linearifolia (c) and Boea hygrometrica (d) 
Nearly $12.9 \%$ of function genes are intron-containing ones, including 10 protein coding genes and 7 tRNA ones, while $\operatorname{clpP}$ and $y c f 3$ genes contain two introns (Additional file 10: Table S2). Furthermore, 253 syntenic loci were identified, including 129 syntenic coding loci and 124 syntenic noncoding loci (Additional file 10: Table S2 and Additional file 11: Table S3).

\section{Sequence divergence and consistency analysis}

After alignment and manual correction of the four cp genomes, a cross-genus consensus cp genome sequence with a length of 155,906 bp was obtained, containing a LSC of 86,371 bp, a SSC of 18,455 bp and two IR copies of 25,540 bp (Fig. 3a). Among 155,906 nucleotides, 144,202
(92.5\%) are conserved among the four cp genomes, while 151,691 nucleotide (97.3\%) are conserved among the three Primulina cp genomes (Additional file 12: File S8). The percentage of conserved sites is significantly higher in IR regions (98.5\% and $99.4 \%$ in the four cp genomes and the three Primulina ones, respectively) than SC regions (89.5\% and 96.3\%), which indicates that intergeneric variation between Primulina and Boea is mainly ascribed to $\mathrm{SC}$ regions.

Furthermore, a total of 622 Con_Islands (the regions containing over 50 continuous conserved sites in a crossgenus consensus cp genome sequence) were identified. The longest one is encoding for partial rrn23 gene in IR regions, with the length of $1145 \mathrm{bp}$ (Fig. 3b). Meanwhile,

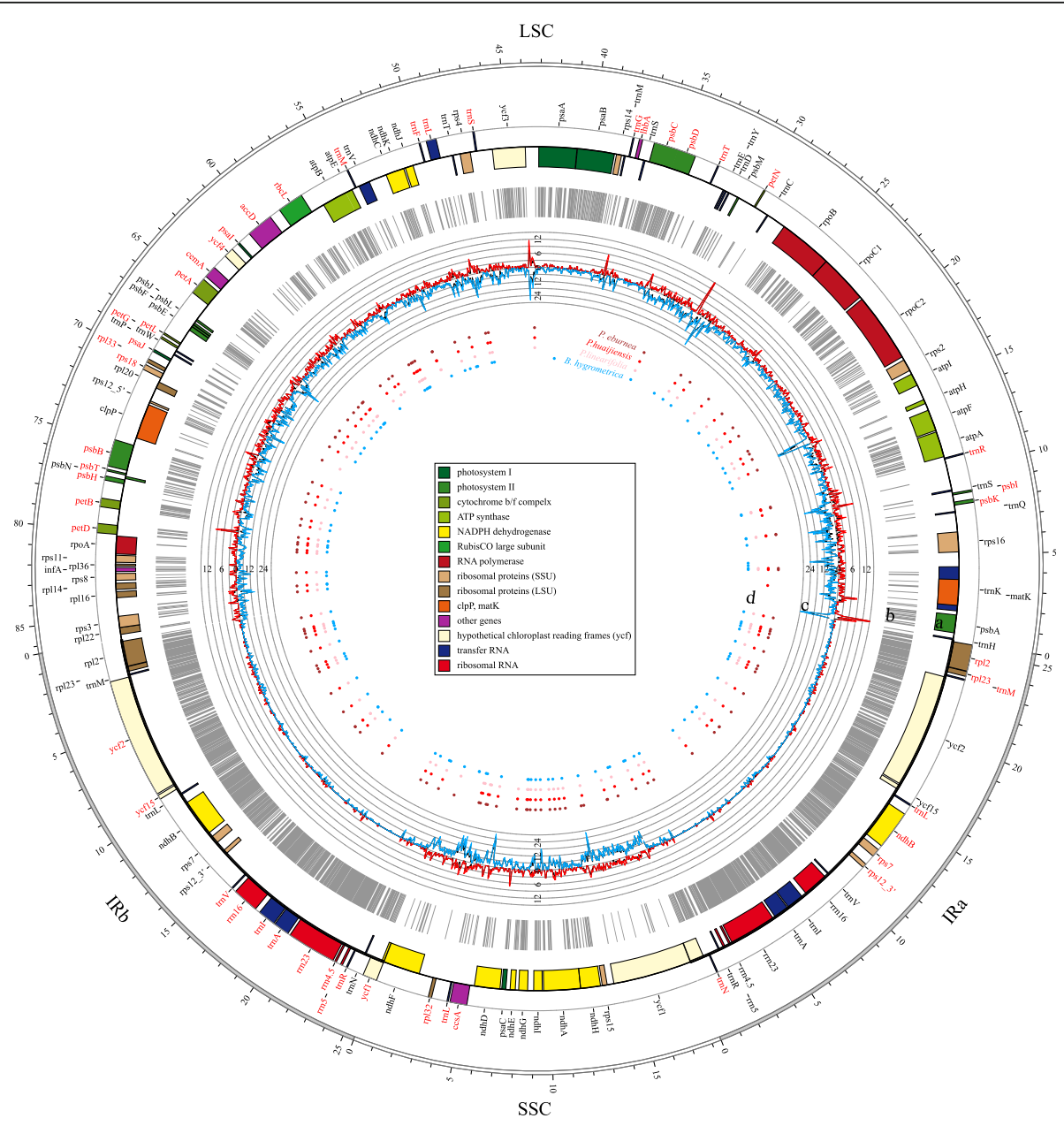

Fig. 3 Circular map of cross-genus consensus chloroplast genome sequences of three Primulina species and Boea hygrometrica. The outermost circle is positions (in Kb) of consensus cp genome sequences. a Annotation of consensus cp genome sequences. Genes shown inside and outside of the circle are transcribed clockwise and counterclockwise, respectively, and their gene names are marked as black and red, respectively. Genes belonging to different groups are marked with different color, with the bar shown in the center. $\mathbf{b}$ Distribution of conserved regions of the four сp genomes. The grey columns represent the Con_Islands, which were defined as the regions containing over 50 continuous conserved sites in cross-genus consensus cp genome sequences. c Distribution of intrageneric and intergeneric polymorphism. The red and blue lines represent average intrageneric and intergeneric PICs in a $100 \mathrm{bp}$ windows with a step of $25 \mathrm{bp}$, respectively. The PICs were counted by the sum of SNP and Indel between two cp genomes. $\mathbf{d}$ Distribution of restriction enzyme site of EcoRl. The outer to inner circles represent the distribution of EcoR I in P. eburnea, P. huaijiensis, P. linearifolia and B. hygrometrica in turns 
622 Con_Seas (the regions between two adjacent Con Islands) were generated, with the longest one of 1409 bp, located at LSC: 62,228-63,636 bp. 181 Con Seas have a length of just $1 \mathrm{bp}$ (Fig. $3 \mathrm{~b}$ and Additional file 13: Table S4). In general, coding regions tend to have longer Con_Islands. Nevertheless, several large Con_Islands were detected in intergenic regions/introns, and partial exons were variant (Fig. 3b).

\section{Determination of high variation regions}

The sliding window analysis revealed that the average intrageneric and intergeneric PICs of all the 6234 windows is 0.82 and 3.51 per $100 \mathrm{bp}$, respectively. The intrageneric and intergeneric PICs of all the windows have a weak positive correlation $(r=0.58 ; p<0.0001$; Additional file 14: Table S5). A total of 18 sliding windows from five regions have intrageneric PIC higher than 9. All of these five regions are located in LSC regions, and the windows with highest PIC (PIC $=19)$ is around $29 \mathrm{~kb}$ in LSC (Fig. 3c and Additional file 14: Table S5). For intergeneric polymorphism, 24 sliding windows from 8 regions have a PIC over 24, with the top PIC of 34.33, located at the position of $15 \mathrm{~kb}$ of LSC. Three high intergeneric regions overlap with high intrageneric regions, appearing at the regions near 0,9 and $44 \mathrm{~kb}$ of LSC, respectively (Fig. 3c and Additional file 14: Table S5).

The PICs of 129 syntenic coding loci were summarized in Additional file 11: Table S3. Almost all the genes have relatively low intrageneric and intergeneric PIC, with average value of 0.46 and 2.11 per $100 \mathrm{bp}$, respectively, therefore they are significantly and strongly correlated $(r=0.99, p<0.0001)$. Nevertheless, an exception was discovered in the longest $y c f 1$ gene $(5.5 \mathrm{~kb})$, which has an intrageneric and intrageneric PIC value of 76.33 and 402.67, respectively. This region has the highest polymorphism per $100 \mathrm{bp}$. While for the 124 syntenic noncoding loci, the average intrageneric and intergeneric polymorphism are 1.30 and 5.52 per $100 \mathrm{bp}$, respectively, and they are also highly correlated $(r=0.88, p<0.0001)$ (Additional file 11: Table S3). In detail, trnS-trnR has both the highest intrageneric and intergeneric PICs, however, its length is much longer than two-directional Sanger sequencing. TrnH-psbA has the second highest intrageneric PIC, but relative lower intergeneric PIC. Another seven regions (trnT-trnL, trnF-ndhJ, trnT-psbD, trnCpet , ndhF-rpl32, psaA-ycf3 and rpl32-trnL) also have relative high intrageneric PICs (Fig. 4a and Additional file 11: Table S3). For intergeneric PICs, rps16-trnQ, atpH-atpI and rpoB-trnC are listed as the top 2-4 highest; however, their intrageneric ones are relative lower. Similar loci were widely observed, such as trnK-rps16, petA-psbJ, rps15-ycf1 and $\operatorname{ccs} A-n d h D$ (Fig. 4b and Additional file 11: Table S3).
The high variation regions identified based on continuous conserved sites (the third region division method) were summarized in Additional file 13: Table S4. The average intrageneric and intergeneric PICs of 622 Con Seas are 1.75 and 7.51 per $100 \mathrm{bp}$, which are significantly higher than the polymorphism of noncoding regions (1.30 and 5.52), respectively. And the correlation coefficient $(r=0.90, p<0.0001)$ is also slightly higher than that of noncoding regions $(\mathrm{r}=0.88)$ (Additional file 13: Table S4). The highest intrageneric polymorphism region is Con_Sea_1, overlapping with trnH-psbA. It is a slightly higher PIC (30) than trnH-psbA (28.67), because partial $p s b A$ mutational sites was added into Con_Sea_1. Among the 601 Con_Seas with length less than 700 bp, Con_Sea_192, overlapping with $p s a A-y c f 3$, is the second highest intrageneric polymorphism region $(\mathrm{PIC}=21.33$ ). This region excludes the front section of $p s a A-y c f 3$ (about $230 \mathrm{bp}$ ), which has low variation (PIC $=0.67$ ), making it possible to be sequenced in a single Sanger reaction. In addition, trnS-trnR was divided into Con_Sea_26 and Con_Sea_27, both of which have relatively high intrageneric polymorphism, with PIC over 20 (Fig. 4c and Additional file 13: Table S4). In addition, several partial ycf1 were identified as high intergeneric polymorphism regions (Fig. 4d and Additional file 13: Table S4).

\section{Evaluation of high variation regions}

The PCR results showed that all the eight newly developed cp primers (Fig. 4c and d) were perfectly amplified in all 49 samples from 44 Primulina species. The levels of polymorphism of the eight markers developed from Con_Seas, compared with the four traditional loci developed from noncoding regions, were summarized in Additional file 15: Table S6. The regions amplified from cp primers of Con_Sea_1 (overlapping with trnH-psbA) had the highest PIC, which is consistent with the prediction by bioinformatics. The PIC ranking of 8 newly developed markers calculated by experimental evaluation are almost the same as that obtained from bioinformatic analysis, with the only difference in the exchange of the ranking of rpoB-trnC (listed as 3rd and 4th based on experiment and bioinformatic methods, respectively) and rpl21-trnL (listed as 4th and 3rd, respectively). Furthermore, the PIC values of 8 novel markers calculated by these two methods are highly correlated $(r=0.85$, $p=0.0075$ ) (Additional file 16: Fig. S2). In addition, consistent with our expectation, all the new markers were observed to have higher polymorphism than traditional ones with the exception for rpl32-trnL, which ranked in the 7th among the 12 loci tested. Interestingly, we found that the rpl32-trnL developed from Con_Seas method ranked higher in polymorphism than the locus developed from traditional noncoding regions (Additional file 15: Table S6). This result indicated that the new primer 

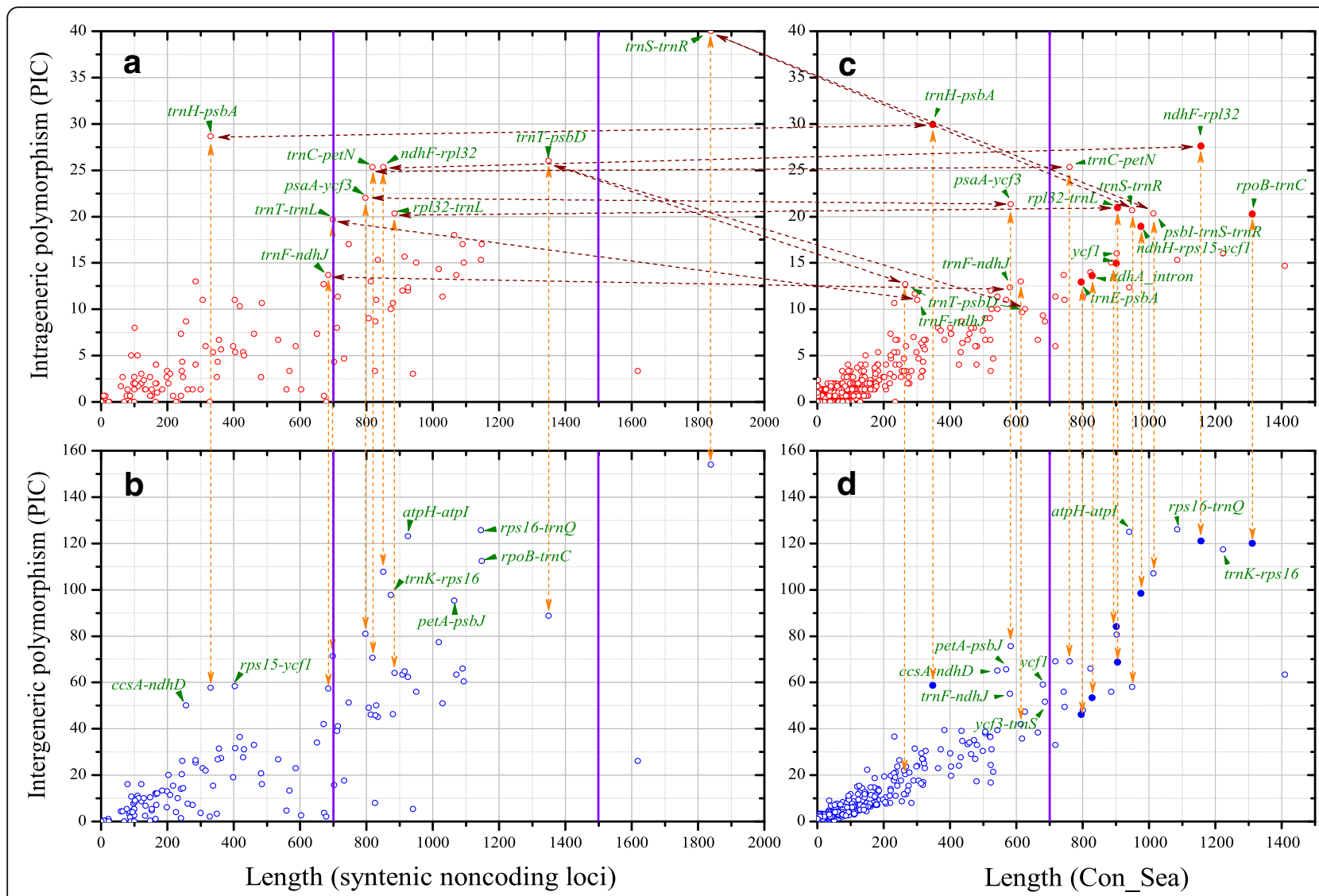

Fig. 4 Intrageneric and intergeneric polymorphism of chloroplast variation regions of three Primulina species and Boea hygrometrica. Intrageneric (a) and intergeneric (b) polymorphism (PICs) of noncoding regions of cp genomes. Intrageneric (c) and intergeneric (d) polymorphism (PICs) of Con_Sea regions of cp genomes. PICs were counted by the sum of SNP and Indel between two cp genomes. Con_Sea is a region between two adjacent Con_Islands, which is defined as the regions containing over 50 continuous conserved sites in cross-genus consensus genome sequences. Eight regions used for experimental evaluation were signed as filled circles, while others were empty circles. The name of the regions with high variation or used for experimental evaluation was marked along the circles. It was noting that the name of these regions in part $C$ and $D$ was shown as the name of noncoding regions overlapped with corresponding Con_Seas, instead of original ID of Con_Seas. The yellow lines link the same regions, while the red lines link the corresponding regions between noncoding regions and Con_Seas

pairs seem to be a better choice for phylogenetic and population studies in Primulina.

\section{Sub-assembly of chloroplast genome from PE RAD-Seq (SACRing)}

Compared to several popular restriction enzyme sites (REs), EcoR I was considered to be suitable for Primulina cp assembly from PE RAD-Seq, for its relative uniform distribution and modest number, which is between 107 and 117 in the 4 complete cp genomes obtained in this study (Fig. 3d). Therefore EcoR I was used in the library construction of 21 individuals, with the number of reads ranging from 145,876 and 797,450. These reads were isolated and used for further sub-assembly.

The average number of RAD tags in CZYX01 ( $P$. eburnea) is 154, slightly higher than that in CZYX02 ( $P$. yongxingensis) (142) and CZYX03 (P. juliae) (144) (Table 2). The percentage of pTs (paired tags, mixtures of two Tag1s at forward and reverse directions of the same restriction enzyme site), oTs (overlap tags, mixtures of Tag1 and its paired Tag2, which was sub-assembled from read2, according to the overlap) and poTs (paired \& overlap tags, mixtures of two paired RAD Tag1s and both of their paired Tag2s) among the three populations is 80 $84 \%, 80-81 \%$, and $59-61 \%$, respectively (Table 2). The length of poT ranged from 734 to $1692 \mathrm{bp}$, with the mean value of $1257 \mathrm{bp}$. Over $93.2 \%$ of poTs have a length over 1000 bp (Fig. 5). The comparative analysis showed that the 20 most conserved poTs were obtained in all the individuals, and they have relative consistent and longer length, $90 \%$ of which have an average length over $1240 \mathrm{bp}$ (Fig. 5). And the consistent sequences of 20 poTs were further assembled into $14 \mathrm{cp}$ fragments, with the total length of 19,536 bp, taking over 15\% of the cp genomes (Fig. 6a and b). The Maximum Likelihood and Bayesian phylogenetic tree showed that these 21 individuals could 


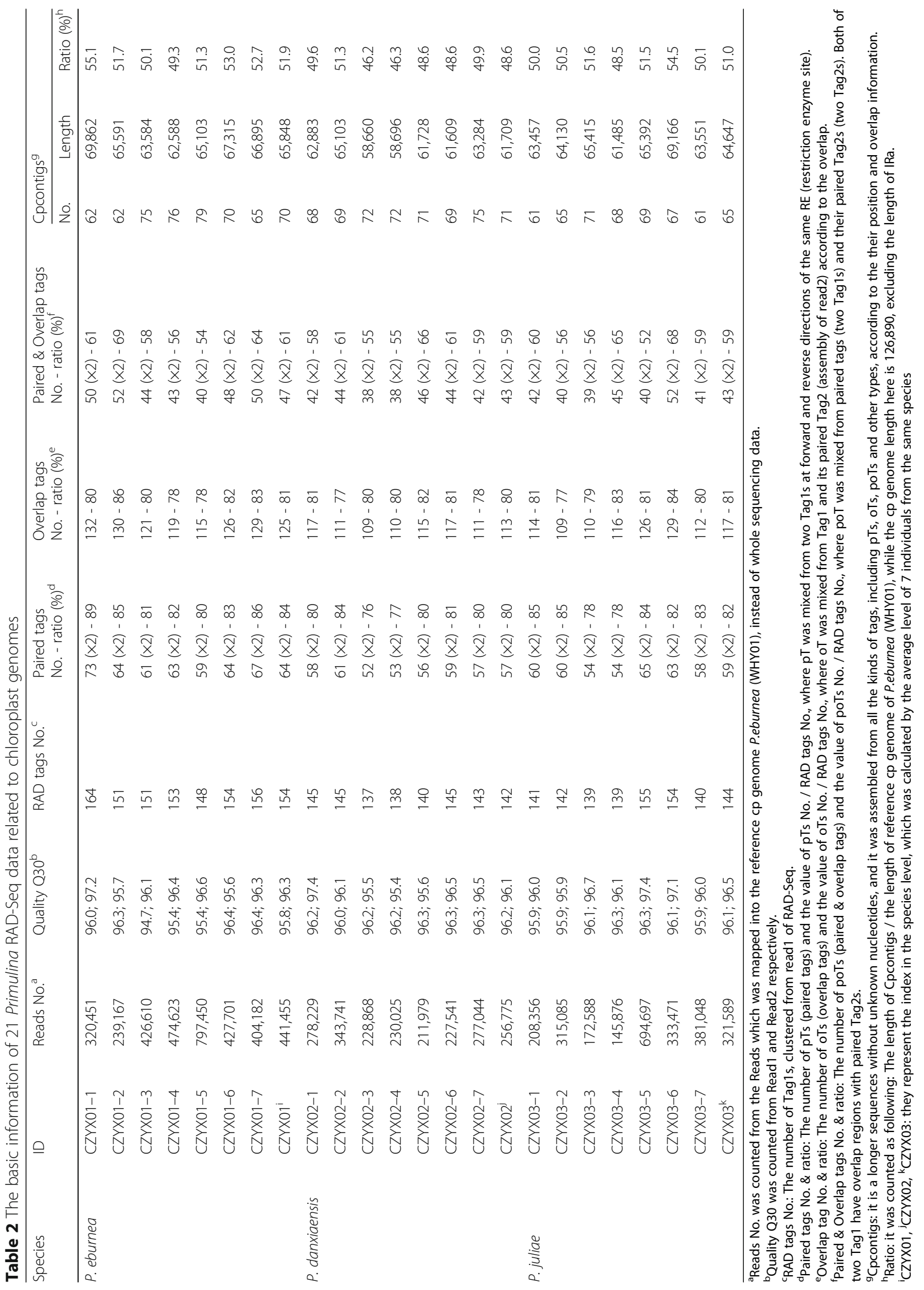




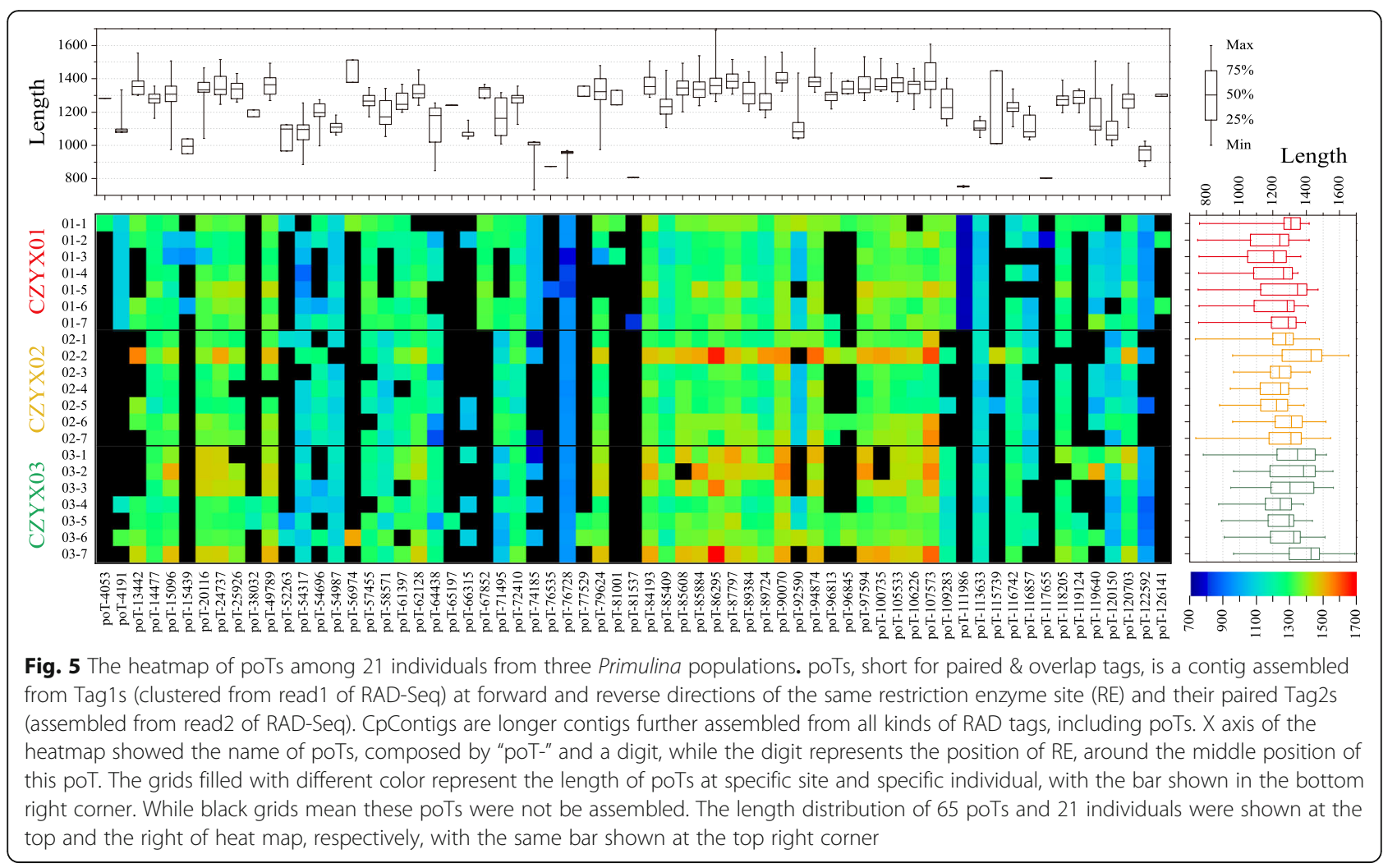

be divided into four groups, with CZYX03 split into two groups (Fig. 6c). Meanwhile, 84 SNP sites were identified from $14 \mathrm{cp}$ fragments among 21 individuals. CZYX03 showed the highest intra-population variation, followed by CZYX02 and CZYX01. The inter-population genetic differentiation between CZYX02 and CZYX03 is significantly lower than that of others (Fig. 6c).

The average number of CpContigs in CZYX01, CZYX02 and CZYX03 is 70, 71 and 65, occupying around $52 \%, 49 \%$ and $51 \%$ of the entire cp genome, respectively (Table 2). Furthermore, 62 consistent CpContigs, which were distributed equably across whole $\mathrm{cp}$ genome, were isolated, with the total length of $42,797 \mathrm{bp}$, accounting for over one third of the cp genome (Fig. 6a and b). A total of 355 SNP were identified from the CpContig, over four folds of that from the poT dataset (84 SNPs). Nevertheless, the results of population genetic analysis of the $\mathrm{cp}$ concatenated sequences from consistent CpContigs are similar to that from consistent poTs (Fig. 6c and d). These results indicated that around half of Primulina cp genome could be directly assembled from RAD-Seq data through our SACRing pipeline. Both poTs and CpContigs could provide enough cp information and variant SNP sites for phylogenetic and population genetic studies.

\section{Discussion}

\section{Lineage-specific high variable regions}

With the rapid development and wide application of NGS technology, it has become much easier to obtain complete chloroplast genomes, as evidenced by the dramatic increase in the number that are publically available (http://www.ncbi.nlm.nih.gov/genome/browse/). Recent comparative plastid genomic studies reveal a pattern of lineage-specific high variable regions in different lineages [3, 7-9]. For example, the most variable regions identified in the genus Pyrus are $n d h C$-trnV, trnR-atpA, $n d h F-r p l 32, p s b M-t r n D$, and trnQ-rps16, while only two (ndhF-rpl32 and trnK-rps16) were consistently found among the Shaw et al., [6] top-ranked 30 cpDNA regions [9]. In this study, the top-9 ranked high variable regions in Primulina overlap with trnH-psbA, ndhF-rpl32, trnC-petN, psaA-ycf3, rpl32-trnL, trnS-trnR, psbI-trnS-trnR, rpoB-trnC and $n d h H-r p s 15-y c f 1$ (Fig. 4c). Of them, only five are listed among the top-ranked 34 cpDNA regions by Shaw et al., [6]. In contrast, the most variable regions identified between the genera Primulina and Boea overlap with rps16trnQ, atpH-atpI, ndhF-rpl32, rpoB-trnC and trnK-rps16 (Fig. 4d), all of which are listed top 14th highest variable regions by Shaw et al., [6]. Our experimental tests confirmed that the new markers developed from Primulina cp 


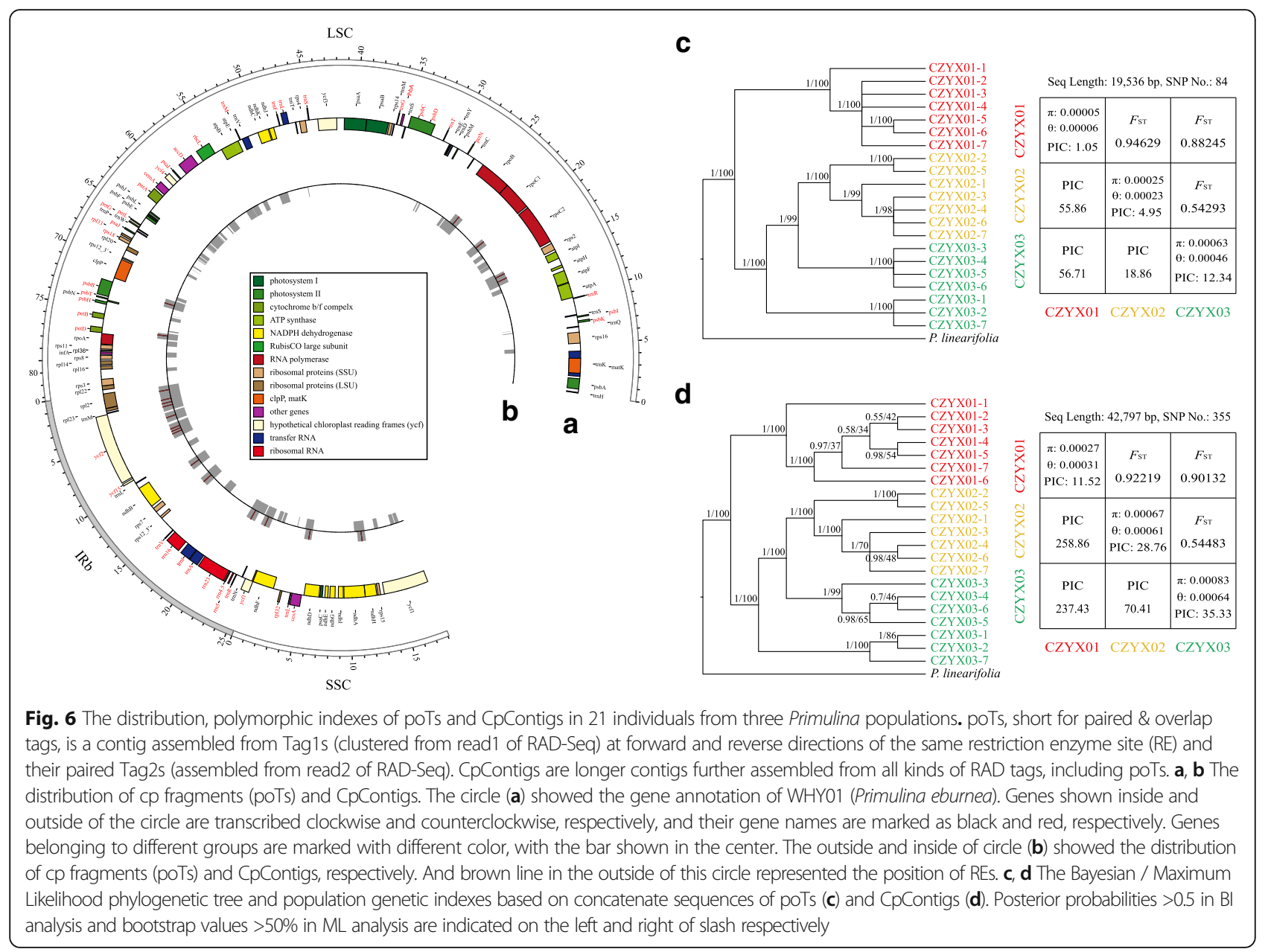

genomes can provide higher polymorphism than the traditional cp primers developed from noncoding regions of distantly related angiosperms. Although listed in top-ranked variable regions, these traditional markers show only moderate variation in Primulina cp genomes (Additional file 15: Table S6). These results imply that the cp markers developed from the three Primulina cp genomes are more suitable in phylogenetic and population studies of Primulina than the traditional and universal cp markers. On the other hand, the makers developed from high variable regions between Primulina and Boea can be used in the higher level phylogeny analysis in the Gesneriaceae family.

\section{A novel strategy for determination of high variation regions: From noncoding regions to Con_Seas regions} Chloroplast DNA markers are usually developed from noncoding regions with high variation $[5,6]$. Here we proposed an improved method to develop cp primers from truly high variation regions (i.e., Con_Seas, the regions between two adjacent conserved regions) across whole cp genomes, instead of noncoding regions. These two methods were further compared systematically and globally. Our newly proposed method has several advantages. First, continuous conserved regions and synthetic coding regions are not matched perfectly, and partial Con_Islands (the conserved regions in cross-genus consensus genome sequences) exist in noncoding regions (Fig. 3a and b). For example, the length of trnS-trnR is too long to be sequenced entirely with two sequencing reactions although this region has both the highest intrageneric and intergeneric PIC (Fig. 4a and b). The trnStrnR region cannot be used for developing markers with the traditional strategy. Since there is a 76 bp Con_Island in the middle of this region (Fig. $3 a$ and b), the trnS-trnR region was divided into two high variation regions according to our Con_Seas method, both of which are suitable for development of cp primers. Second, long functional genes usually have many mutation hotspot regions. For example, the 5.5-kb long $y c f 1$ gene has nine Con_Seas but no introns, and several Con_Seas have high mutational hotspots, especially in intergeneric polymorphism (Fig. 3a, b and c). These regions were ignored in previous marker development; however, several highly 
variable regions were identified in our method (Fig. 4c and $\mathrm{d}$ ), and the polymorphism of one locus has been verified experimentally (Additional file 15: Table S6). Third and similarly, the boundaries of coding regions of functional genes (or even the entire coding regions) are not always conserved enough to develop perfect $\mathrm{cP}$ primers, especially in SSC region (Fig. 3a and b). Therefore, it is difficult for developing suitable markers using traditional methods, while our method could avoid this problem to a remarkable extent.

In this study, 18 Con_Seas have both intrageneric and intergeneric PICs higher than that of Con_Sea_124 $(\operatorname{trn} E-\operatorname{trn} T)$, which is 9th most variable in our experimental evaluation (Additional file 13: Table S4 and Additional file 15: Table S6). The highly variable nature of these loci will greatly assist phylogenetic studies in Primulina. We believe that this strategy for determination of high variation regions based on Con_Seas would provide a reference for development of high polymorphic cp markers of other plants with whole $\mathrm{cp}$ genomes. Alignment of two plastomes could be supported by our software "Con_Sea_Identification_and_PIC_ Calculation", but the result may have a poor positive correlation with the true variability across the taxon of interest if only two taxa are used. We recommended that at least three cp genomes be available when using it. It was noteworthy that this strategy and software needs to be continually optimized by any user, to determine setting how to select the best parameter of minimum length of Con_Island. The adjacent Con_Seas with relative short length could be joined up as a new region, in order to provide the most variable characters in one or two Sanger sequencing reactions.

\section{A novel strategy to generate $\mathrm{cp}$ sequences for phylogenetic studies: From sanger sequencing to PE RAD-Seq}

RAD-Seq is increasingly used in population genetic and phylogenetic studies, due to the rapidly decreasing cost of sequencing [47]. However, only nuclear genetic information is typically extracted and analyzed. In this study, we have developed a novel strategy to obtain large amount of cp variable characters directly from RAD-Seq. Using our pipeline SACRing, around $44 \mathrm{cp}$ sequences with the average length of $1260 \mathrm{bp}$, and 64-kb-long CpContigs (sub-supermarker, half of whole cp genome) were obtained from RAD-Seq of the 21 Primulina individuals, which we used for population genetic analyses (Table 2 and Fig. 5). We believe that having publically available tools to handle $\mathrm{cp}$ data in RAD-Seq datasets could allow those working with nuclear RAD-Seq datasets to enrich them with cp genome data at no further cost, greatly increasing their value.

Complete cp genome sequences were recently proposed as super marker for DNA barcoding of plants, which could greatly improve resolution [20, 48].
However, recent studies revealed that such a supermarker may not substantially improve discrimination of clades that recently diverged or that have complex patterns of hybridization [49-51]. Furthermore, it is still not easy for researchers without programming background to isolate and assemble cp genomes from NGS data. In addition, many experimental aids or manual corrections are required in the steps of gap closing and annotation. Therefore, complete cp genomes for dozens or hundreds of individuals is still impractical in most studies. Using the pipeline SACRing, a sub-super-marker (CpContigs), comprising c. 50\% of entire cp genomes, could be easily obtained for hundreds of individuals in a fully automated approach and in a relative short amount of time. Compared with super-markers (i.e., entire cp genomes, which was assembled from genome survey sequencing), our sub-super-marker (CpContigs) or even poTs could provide sufficient variable characters but with lower cost. RAD-Seq with specific restriction enzyme site could obtain enough raw NGS data for cp genome assembly at a much lower sequencing throughput, c. $5-10 \%$ of that of genome survey sequencing.

On the other hand, some previous studies have used resequencing (mapping the NGS reads to the reference cp genomes directly) to call the SNPs of cp DNA [52]. However, such direct mapping approaches lead to errors of intra-individual polymorphism because of DNA transfer of cp sequences into the nuclear or mitochondrial genomes or the both [53]. In our strategy, the assembly of poTs or CpContigs seem to be accurate due to the high abundance of chloroplast genomes, which are 1-2 orders of magnitude of mitochondrial genome, and 2-4 orders of magnitude of nuclear genome in a single leaf cell [52]. Because variable characters were identified based on cluster results of poTs or CpContigs, candidate assembly errors could be identified. Therefore, our strategy could minimize spurious intra-individual polymorphism.

To the best of our knowledge, this study is the first to develop the sub-assembly of poTs and CpContigs (subsuper-marker, c. $50 \%$ of entire cp genomes) from $\mathrm{PE}$ RAD-Seq, and the SACRing was the first pipeline to bridge the relationship between RAD-Seq and cp genomes. The pipeline can be download from https:// github.com/scbgfengchao/, where it will be subjected to continual improved and updated. This strategy expands the application of RAD-Seq, and would be practice for large-scale application of poTs or sub-super-marker in phylogenetic and population genetic studies.

We recommend that in the cases where only dozens of variable sites are required and where at least $3 \mathrm{cp}$ genomes of related species are available, the first strategy "from noncoding regions to Con_Seas regions" will be more effective. Otherwise, our second strategy is 
recommended, particularly when only a single $\mathrm{cp}$ genome of closely related species is available. We also recommend the second method when the aim is to supplement a population genetics study using RAD-seq at nuclear loci with cp loci.

\section{Conclusions}

In this study, three complete Primulina chloroplast genomes were assembled from genome survey data. Combined with the cp genome of Boea hygrometrica, several lineage-specific highly variable cp markers were developed from the true high variation regions (Con_Seas) across whole cp genomes using the software "Con_Sea_Identification_and_PIC_Calculation". This approach provided higher polymorphism than traditional cp primers, which was confirmed by experimental evaluation results. The newly developed markers will promote phylogenetic and population genetic studies in Primulina and other genera of the family Gesneriaceae.

We also wrote a novel Bash script, SACRing, which uses RAD-Seq data to directly assemble cp fragments (poTs) and sub-super-marker (CpContigs), representing around half of the completed cp genome (in the case of 21 individuals from three Primulina populations). The conserved poTs or CpContigs could be further applied in the studies of population genetic analysis and phylogenetics. Our method fundamentally alters traditional approaches, which have been deeply dependent on large amounts of Sanger sequencing based on cp primers. These two novel strategies proposed in our study may provide a reference to similar research in other non-model species fascinated by next generation sequencing.

\section{Additional files}

Additional file 1: Table S1. The list of 49 individuals from 44 Primulina species used for evaluation of the polymorphism of chloroplast markers and GenBank accession id of $12 \mathrm{cp}$ sequences for each individual (XLSX $16 \mathrm{~kb}$ )

Additional file 2: File S1. The script of QC (quality control of Illumina PE reads) (ZIP $4 \mathrm{~kb}$ )

Additional file 3: File S2. The script of Correspondence_between_ each_seq_and_consensus (ZIP 6 kb)

Additional file 4: File S3. The script of Con_Sea_Identification_and_ PIC_Calcalation (ZIP $182 \mathrm{~kb}$ )

Additional file 5: File S4. The script of PIC_calculation (Calculation of SNP No., InDel No. and PIC values between two sequences after alignment) (ZIP $14 \mathrm{~kb}$ )

Additional file 6: File S5. The script of SACRing (Sub-Assembly of Chloroplast genome from PE RAD-seq) (ZIP $31264 \mathrm{~kb}$ )

Additional file 7: File S6. The script of RAD_QC_pe (Quality Control of PE RAD-seq) (ZIP $5 \mathrm{~kb}$ )

Additional file 8: Figure S1. The mapping depth and coverage of three Primulina chloroplast genomes shown in Integrative Genomics Viewer, P. eburnea (a), P. huaijiensis (b), P. linearifolia (c) (PDF 819 kb)
Additional file 9: File S7. The gff3 files of $P$. eburnea, P. huaijiensis, $P$. linearifolia and B. hygrometrica (ZIP $22 \mathrm{~kb}$ )

Additional file 10: Table S2. Feature of function genes and syntenic coding/noncoding loci of four chloroplast genomes and consensus sequences (XLSX $406 \mathrm{~kb}$ )

Additional file 11: Table S3. Intrageneric and Intergeneric polymorphism of each syntenic coding loci and syntenic noncoding loci (XLSX $41 \mathrm{~kb}$ )

Additional file 12: File S8. The cluster result of four chloroplast genomes of $P$. eburnea, P. huaijiensis, P. linearifolia and B. hygrometrica (FAS $609 \mathrm{~kb}$ )

Additional file 13: Table S4. Intrageneric and Intergeneric polymorphism of each Con_Sea region (XLSX $73 \mathrm{~kb}$ )

Additional file 14: Table S5. Intrageneric and Intergeneric polymorphism of each window region (XLSX $583 \mathrm{~kb}$ )

Additional file 15: Table S6. The bioinformatic analysis and experimental evaluation of eight newly developed chloroplast markers and four traditional ones (XLSX $12 \mathrm{~kb}$ )

Additional file 16: Figure S2. The PIC values of eight newly developed chloroplast markers calculated by bioinformatic analysis and experimental evaluation (PDF $222 \mathrm{~kb}$ )

\section{Abbreviations}

Con_Islands: regions containing over 50 continuous conserved sites in the cross-genus consensus genome sequences; Con_Seas: the regions between two adjacent Con_Island; cp: chloroplast; IR: inverted repeat; ITS: nuclear ribosomal internal transcribed spacer; LSC: large single copy; NGS: nextgeneration sequencing; oTs: overlap tags; PE: paired-end; PIC: potentially informative characters; poTs: paired \& overlap tags; pTs: paired tags; RAD-Seq: restriction-site associated DNA sequencing; SACRing: Sub-assembly of chloroplast from PE RAD-Seq; SSC: small single copy

\section{Funding}

This work was supported by National Natural Science Foundation of China (U1501211, 31501799).

\section{Availability of data and materials}

The chloroplast sequencing data of P. linearifolia (GXNN01), P. huaijiensis (GDHJ02), P. eburnea (WHY01) and 21 Primulina individuals from three natural populations (CZYX01, P. eburnea; CZYX02, P. yongxingensis; CZYX03, P. juliae), was deposited in GenBank with accession of SRR4001599, and SRR4001604 - SRR 4001608, respectively. And three completed Primulina cp genomes and 21 sub-super-markers are also submitted to GenBank with accession of.

MF472011 - MF472013, and MF480524 - MF480544, respectively. Besides, the GenBank accession of 8 newly developed cp primers and 1 traditional primers for 49 Primulina samples are MF472014 - MF472454, with the detailed correspondence shown in additional file 1.

\section{Authors' contributions}

This study was conceived by MK and CaF. Collection and identification of field material was performed by MK. Sample preparation, nuclei isolation, and PCR analyses were performed by MX and CeF. Data analysis was conducted by CaF. $\mathrm{CaF}, \mathrm{MK}$ and EV wrote the paper. All authors read and approved the final manuscript.

Ethics approval and consent to participate

Not applicable.

\section{Consent for publication}

Not applicable.

\section{Competing interests}

The authors declare that they have no competing interests.

\section{Author details}

${ }^{1}$ Key Laboratory of Plant Resources Conservation and Sustainable Utilization, South China Botanical Garden, Chinese Academy of Sciences, 723 Xingke Road, Guangzhou 510650, China. ${ }^{2}$ University of Chinese Academy of 
Sciences, Beijing 100049, China. ${ }^{3}$ Department of Plant and Soil Sciences, University of Vermont, Burlington, VT 05405, USA.

\section{Received: 30 November 2016 Accepted: 31 October 2017 Published online: 07 November 2017}

\section{References}

1. Powell W, Morgante M, Mcdevitt R, Vendramin GG, Rafalski JA. Polymorphic simple sequence repeat regions in chloroplast genomes - applications to the population-genetics of pines. Proc Natl Acad Sci U S A. 1995;92:7759-63.

2. Petit RJ, Vendramin GG. Plant phylogeography based on organelle genes: an introduction. Phylogeography of Southern European Refugia. 2007:23-97.

3. Zhang YJ, Ma PF, Li DZ. (2011). High-throughput sequencing of six bamboo chloroplast genomes: phylogenetic implications for temperate woody bamboos (Poaceae: Bambusoideae). PLoS One 2011;6:e20596.

4. Yang YC, Zhou T, Duan D, Yang J, Feng L, Zhao GF. Comparative analysis of the complete chloroplast genomes of five Quercus species. Front Plant Sci. 2016;7:959.

5. Shaw J, Lickey EB, Beck JT, Farmer SB, Liu WS, Miller J, et al. The tortoise and the hare II: relative utility of 21 noncoding chloroplast DNA sequences for phylogenetic analysis. Am J Bot. 2005;92:142-66.

6. Shaw J, Lickey EB, Schilling EE, Small RL. Comparison of whole chloroplast genome sequences to choose noncoding regions for phylogenetic studies in angiosperms: the tortoise and the hare III. Am J Bot. 2007;94:275-88.

7. $\quad$ Dong WP, Liu J, Yu J, Wang L, Zhou SL. Highly variable chloroplast marker for evaluating plant phylogeny at low taxonomic levels and for DNA barcoding. PLoS One. 2012:7:e35071.

8. Sarkinen T, George M. Plastid marker variation: can complete plastid genomes from closely related species help? PLoS One. 2013;8:e82266.

9. Korotkova N, Nauheimer L, Ter-Voskanyan H, Allgaier M, Borsch T. Variability among the most rapidly evolving plastid genomic regions is lineagespecific: implications of pairwise genome comparisons in Pyrus (Rosaceae) and other angiosperms for marker choice. PLoS One. 2014;9:e112998.

10. Choi KS, Chung MG, Park S. The complete chloroplast genome sequences of three Veroniceae species (Plantaginaceae): comparative analysis and highly divergent regions. Front Plant Sci. 2016;7:355.

11. Prince LM. Plastid primers for angiosperm phylogenetics and phylogeography. Appl. Plant Sci. 2015:3:1400085.

12. Straub SCK, Fishbein M, Livshultz T, Foster Z, Parks M, Weitemier K, et al. Building a model: developing genomic resources for common milkweed (Asclepias syriaca) with low coverage genome sequencing. BMC Genomics. 2011;12:211.

13. Egan AN, Schlueter J, Spooner DM. Applications of next-generation sequencing in plant biology. Am J Bot. 2012;99:175-85.

14. Harrison N, Harrison RJ, Kidner CA. Comparative analysis of Begonia plastid genomes and their utility for species-level phylogenetics. PLoS One. 2016; 11:e0153248.

15. Yang JB, Li DZ, Li HT. Highly effective sequencing whole chloroplast genomes of angiosperms by nine novel universal primer pairs. Mol Ecol Resour. 2014;14:1024-31

16. Mariac C, Scarcelli N, Pouzadou J, Barnaud A, Billot C, Faye A, et al. Costeffective enrichment hybridization capture of chloroplast genomes at deep multiplexing levels for population genetics and phylogeography studies. Mol Ecol Resour. 2014;14:1103-13.

17. Dong WP, Liu H, Xu C, Zuo YJ, Chen ZJ, Zhou SLA. Chloroplast genomic strategy for designing taxon specific DNA mini-barcodes: a case study on ginsengs. BMC Genet. 2014;15:138.

18. Baird NA, Etter PD, Atwood TS, Currey MC, Shiver AL, Lewis ZA, et al. Rapid SNP discovery and genetic mapping using sequenced RAD markers. PLoS One. 2008;3:e3376

19. Moore MJ, Dhingra A, Soltis PS, Shaw R, Farmerie WG, Folta KM, et al. Rapid and accurate pyrosequencing of angiosperm plastid genomes. BMC Plant Biol. 2006;6:17

20. Parks M, Cronn R, Liston A. Increasing phylogenetic resolution at low taxonomic levels using massively parallel sequencing of chloroplast genomes. BMC Biol. 2009;7:84.

21. Njuguna W, Liston A, Cronn R, Ashman TL, Bassil N. Insights into phylogeny, sex function and age of Fragaria based on whole chloroplast genome sequencing. Mol Phylogenet Evol. 2013;66:17-29.

22. Wei YG. Gesneriaceae of South China: Guangxi Science and Technology Publishing House; 2010.
23. Ai B, Gao Y, Zhang XL, Tao JJ, Kang M, Huang HW. Comparative transcriptome resources of eleven Primulina species, a group of 'stone plants' from a biodiversity hot spot. Mol Ecol Resour. 2015;15:619-32.

24. Hao Z, Kuang YW, Kang M. Untangling the influence of phylogeny, soil and climate on leaf element concentrations in a biodiversity hotspot. Funct Ecol. 2015:29:165-76.

25. Wang YZ, Mao RB, Liu Y, Li JM, Dong Y, Li ZY, et al. Phylogenetic reconstruction of Chirita and allies (Gesneriaceae) with taxonomic treatments. J Syst Evol. 2011:49:50-64.

26. Weber A, Middleton DJ, Forrest A, Kiew R, Lim CL, Rafidah AR, et al. Molecular systematics and remodelling of Chirita and associated genera (Gesneriaceae). Taxon. 2011;60:767-90.

27. Kang M, Tao JJ, Wang J, Ren C, Qi QW, Xiang QY, et al. Adaptive and nonadaptive genome size evolution in karst endemic flora of China. New Phytol. 2014;202:1371-81.

28. Gao Y, Ai B, Kong HH, Kang M, Huang HW. Geographical pattern of isolation and diversification in karst habitat islands: a case study in the Primulina eburnea Complex. J Biogeogr. 2015;42:2131-44.

29. Langmead B, Salzberg SL. Fast gapped-read alignment with bowtie 2. Nat Methods. 2012;9:357-U354

30. Zhang TW, Fang YJ, Wang XM, Deng X, Zhang XW, SN H, et al. The complete chloroplast and mitochondrial genome sequences of Boea hygrometrica: insights into the evolution of plant organellar genomes. PLoS One. 2012;7:e30531.

31. Zerbino DR, Birney E. Velvet: algorithms for de novo short read assembly using de Bruijn graphs. Genome Res. 2008;18:821-9.

32. Boetzer M, Pirovano W. SSPACE-LongRead: scaffolding bacterial draft genomes using long read sequence information. BMC Bioinformatics. 2014;15:211.

33. Kent WJ. BLAT - The BLAST-like alignment tools. Genome Res. 2002;4:656-64.

34. Li H, Handsaker B, Wysoker A, Fennell T, Ruan J, Homer N, et al. The sequence alignment/map format and SAMtools. Bioinformatics. 2009; 25:2078-9.

35. Robinson JT, Thorvaldsdottir H, Winckler W, Guttman M, Lander ES, Getz G, et al. Integrative genomics viewer. Nat Biotechnol. 2011;29:24-6.

36. Wyman SK, Jansen RK, Boore JL. Automatic annotation of organellar genomes with DOGMA. Bioinformatics. 2004;20:3252-5.

37. Lowe TM, Eddy SR. tRNAscan-SE: a program for improved detection of transfer RNA genes in genomic sequence. Nucleic Acids Res. 1997;25:955-64.

38. Lohse M, Drechsel O, Kahlau S, Bock R. OrganellarGenomeDRAW-a suite of tools for generating physical maps of plastid and mitochondrial genomes and visualizing expression data sets. Nucleic Acids Res. 2013;41:W575-81.

39. Krzywinski M, Schein J, Birol I, Connors J, Gascoyne R, Horsman D, et al. Circos: an information aesthetic for comparative genomics. Genome Res. 2009;19:1639-45.

40. Li H, Durbin R. Fast and accurate short read alignment with burrowswheeler transform. Bioinformatics. 2009:25:1754-60.

41. Catchen JM, Amores A, Hohenlohe P, Cresko W, Postlethwait JH. Stacks: building and genotyping loci de novo from short-read sequences. G3 (Bethesda). 2011;1:171-82.

42. Huang XQ, Madan A. CAP3: A DNA sequence assembly program. Genome Res. 1999;9:868-77.

43. Darriba D, Taboada GL, Doallo R, Posada D. jModelTest 2: more models, new heuristics and parallel computing. Nat Methods. 2012;8:772.

44. Ronquist F, Huelsenbeck JP. MrBayes 3: Bayesian phylogenetic inference under mixed models. Bioinformatics. 2003;12:1572-4.

45. Stamatakis A. RAxML version 8: a tool for phylogenetic analysis and postanalysis of large phylogenies. Bioinformatics. 2014;9:1312-3.

46. Librado P, Rozas J. DnaSP v5: a software for comprehensive analysis of DNA polymorphism data. Bioinformatics. 2009;25:1451-2.

47. Twyford AD, Ennos RA. Next-generation sequencing as a tool for plant ecology and evolution. Plant Ecol. Divers. 2012;5:411-3.

48. Nock CJ, Waters DLE, Edwards MA, Bowen SG, Rice N, Cordeiro GM, et al. Chloroplast genome sequences from total DNA for plant identification. Plant Biotechnol J. 2011;9:328-33.

49. Fazekas AJ, Kesanakurti PR, Burgess KS, Percy DM, Graham SW, Barrett SCH, et al. Are plant species inherently harder to discriminate than animal species using DNA barcoding markers? Mol Ecol Resour. 2009:9:130-9.

50. Hollingsworth PM, Graham SW, Little DP. Choosing and using a plant DNA barcode. PLoS One. 2011:6:e19254.

51. Ruhsam M, Rai HS, Mathews S, Ross TG, Graham SW, Raubeson LA, et al Does complete plastid genome sequencing improve species discrimination and phylogenetic resolution in Araucaria? Mol Ecol Resour. 2015;15:1067-78. 
52. Sabir JSM, Arasappan D, Bahieldin A, Abo-Aba S, Bafeel S, Zari TA, et al. Whole mitochondrial and plastid genome SNP analysis of nine date palm cultivars reveals plastid heteroplasmy and close phylogenetic relationships among cultivars. PLoS One. 2014;9:e94158.

53. Scarcelli N, Mariac C, Couvreur TLP, Faye A, Richard D, Sabot F, et al. Intraindividual polymorphism in chloroplasts from NGS data: where does it come from and how to handle it? Mol Ecol Resour. 2016;2:434-45.

Submit your next manuscript to BioMed Central and we will help you at every step:

- We accept pre-submission inquiries

- Our selector tool helps you to find the most relevant journal

- We provide round the clock customer support

- Convenient online submission

- Thorough peer review

- Inclusion in PubMed and all major indexing services

- Maximum visibility for your research

Submit your manuscript at www.biomedcentral.com/submit
Biomed Central 